\title{
The release of the adipocytokine visfatin is regulated by glucose and insulin
}

\author{
D. G. Haider • G. Schaller • S. Kapiotis • C. Maier • \\ A. Luger • M. Wolzt
}

Received: 2 February 2006 / Accepted: 6 April 2006 / Published online: 31 May 2006

(C) Springer-Verlag 2006

\begin{abstract}
Aims/hypothesis The novel insulin-mimetic adipocytokine visfatin has been linked to the metabolic syndrome, but its regulation has not been characterised to date. Since insulinmimetic actions of visfatin may be part of the feedback regulation of glucose homeostasis, we hypothesised that visfatin concentrations are influenced by glucose or insulin blood levels in humans.

Subjects, materials and methods In this randomised, doubleblind, placebo-controlled crossover study, nine healthy male subjects (age $26 \pm 6$ years) attended three different study days. On each day, systemic glucose concentrations of 5.0, 8.3 and $11.1 \mathrm{mmol} / \mathrm{l}$ were attained by stepwise increases in i.v. infusions of glucose, representing fasting and postprandial conditions. Visfatin plasma concentrations were studied during concomitant exogenous hyperinsulinaemia, inhibition of endogenous insulin production by somatostatin infusion, and placebo time control. Additionally, human adipocytes were cultured to study visfatin release and mRNA expression in vitro.
\end{abstract}

D. G. Haider $\cdot$ G. Schaller $\cdot$ M. Wolzt

Department of Clinical Pharmacology,

Medical University of Vienna,

Währinger Gürtel 18-20,

A-1090 Vienna, Austria

\section{S. Kapiotis}

Institute for Medical and Chemical Laboratory Diagnostics,

Medical University of Vienna,

Vienna, Austria

C. Maier $\cdot$ A. Luger $\cdot$ M. Wolzt $(\bowtie)$

Division of Endocrinology and Metabolism,

Department of Internal Medicine III,

Medical University of Vienna,

Vienna, Austria

e-mail: michael.wolzt@meduniwien.ac.at
Results Glucose concentrations of 8.3 and $11.1 \mathrm{mmol} / 1$ increased circulating visfatin from baseline concentrations of $0.5 \pm 0.0 \mathrm{ng} / \mathrm{ml}$ to $0.9 \pm 0.1$ and $2.1 \pm 0.3 \mathrm{ng} / \mathrm{ml}$, respectively $(p<0.01)$. Glucose-induced elevation of visfatin was prevented by co-infusion of insulin or somatostatin $(p<0.05)$. Cultured subcutaneous and visceral adipocytes released an equivalent amount of visfatin upon glucose-concentrationand time-dependent stimulation. Visfatin secretion involved the phosphatidylinositol 3-kinase (PI3-kinase) and protein kinase B (AKT) pathways. The mRNA expression pattern of visfatin was consistent with this altered protein release. Conclusions/interpretation Circulating visfatin concentrations are increased by hyperglycaemia. This effect is suppressed by exogenous hyperinsulinaemia or somatostatin infusion. Glucose signalling for visfatin release in adipocytes involves the PI3-kinase/AKT pathway.

Keywords Adipocytes · Clamp · Insulin · Randomised controlled trial $\cdot$ Somatostatin $\cdot$ Visfatin

\author{
Abbreviations \\ AKT protein kinase $\mathrm{B}$ \\ DMEM/F12 DMEM/Nutrient Mix F12 \\ PI3-kinase phosphatidylinositol 3-kinase
}

\section{Introduction}

The adipose tissue acts as an endocrine organ and produces several hormones, including leptin [1], adiponectin [2-4], resistin [5] and the recently described visfatin/ pre-B cell colony-enhancing factor [6]. Visfatin is abundantly produced by adipocytes and may link adipositas with diabetes because of its insulin-mimetic action, which is 
Table 1 Subject characteristics

\begin{tabular}{lc}
\hline Age (years) & $26 \pm 6$ \\
BMI $\left(\mathrm{kg} / \mathrm{m}^{2}\right)$ & $23 \pm 1$ \\
Fasting glucose $(\mathrm{mmol} / \mathrm{l})$ & $4.8 \pm 0.1$ \\
Fasting insulin $(\mu \mathrm{U} / \mathrm{l})$ & $7.3 \pm 1.0$ \\
\hline
\end{tabular}

Values are means $\pm \mathrm{SD}, n=9$

exerted by binding to the insulin receptor [6]. This action has been demonstrated in cultured cells and in animal experiments [6].

The mechanisms controlling cellular visfatin secretion have not yet been characterised. Circulating visfatin concentrations were found to be correlated with the amount of visceral fat in healthy non-obese humans [6], consistent with the finding that increased visfatin release and expression is associated with obesity and diabetes in this tissue [7, 8]. Further, increased plasma visfatin concentrations were reduced after weight loss in morbidly obese subjects [9]. Since insulin-mimetic actions of visfatin may be part of the feedback regulation of glucose homeostasis we hypothesised that visfatin concentrations are influenced by glucose or insulin blood levels in humans. We tested this hypothesis by employing glucose clamp experiments to represent fasting and postprandial states with concomitant hyperinsulinaemia during inhibition of endogenous insulin production by somatostatin, and during vehicle control in a double-blind, placebo-controlled three-way crossover trial. In additional experiments, human adipocytes were isolated from subcutaneous and visceral fat and exposed to glucose and insulin to detect dose- or time-dependencies of visfatin protein release and mRNA expression and to assess signalling pathways involved.

\section{Subjects, materials and methods}

\section{Ethical approval}

The study protocol was approved by the Ethics Committee of the University of Vienna and complies with the Declaration of Helsinki, including current revisions, and the European Good Clinical Practice Guidelines. Written informed consent was obtained from all healthy volunteers and tissue sample donors before enrolment

\section{Subjects}

Nine healthy male non-smokers and drug-free volunteers were included in this study (Table 1). A complete health examination including laboratory status within 14 days before the first study day revealed no clinically relevant abnormalities. All subjects claimed not to have ingested any prescribed medications or over-the-counter drugs from 2 weeks before screening until the study was complete. Experiments started after overnight fasting and were conducted in a quiet room with an ambient temperature of $22^{\circ} \mathrm{C}$.

Experimental protocol

The study was designed as a double-blind, placebocontrolled three-way crossover trial. Details of the experimental procedure have been published previously [10]. On three different study days subjects received infusions of insulin, somatostatin or placebo with a washout phase of at least 2 days. This was accompanied by a co-infusion of glucose to achieve stepwise increased venous glucose concentrations of 5.0, 8.3 and $11.1 \mathrm{mmol} / \mathrm{l}$ for a period of $90 \mathrm{~min}$ each on all three study days.

Intravenous cannulas were inserted into opposite arms for blood sampling and drug infusions, respectively. Baseline blood samples were drawn, and infusions were started and maintained for $270 \mathrm{~min}$, these being (1) insulin mixed with heparinised blood (to avoid adsorption to the syringe and to connecting tubing) [11] at $1.0 \mathrm{mU} \mathrm{kg}^{-1}$ $\min ^{-1}$ (Huminsulin; Lilly, Fegersheim, France); (2) somatostatin at $61.1 \mathrm{pmol} \mathrm{kg} \mathrm{min}^{-1}$ (UCB Pharma, Vienna, Austria); or (3) placebo ( $0.9 \%$ saline). Blood glucose concentrations were determined every $5 \mathrm{~min}$ throughout the study (Glucose Analyzer; Beckman, Fullerton, CA, USA), and glucose infusion rates (10\% glucose; Mayerhofer Pharmazeutika, Linz, Austria) were adjusted accordingly.

Laboratory parameters

Blood samples for quantification of venous visfatin and insulin were drawn at baseline and every $90 \mathrm{~min}$. Visfatin was analysed using a commercially available kit (Phoenix Peptides, Karlsruhe, Germany). The limit of detection was $0.1 \mathrm{ng} / \mathrm{ml}$ and the intra-assay CV was 5.6 and $5.8 \%$ for low and high visfatin concentrations, respectively. Laboratory analyses were carried out according to standard procedures at the Department of Medical and Chemical Laboratory Diagnostics, Vienna, Austria.

\section{Cell culture experiments}

Subcutaneous fat was obtained from three lean subjects (one male, two female, $36 \pm 5$ years; BMI $23 \pm 2 \mathrm{~kg} / \mathrm{m}^{2}$; fasting glucose $5.1 \pm 0.4 \mathrm{mmol} / \mathrm{l}$ ) and subcutaneous and visceral fat from seven morbidly obese patients (six female, one male; $47 \pm 13$ years; BMI $47 \pm 5 \mathrm{~kg} / \mathrm{m}^{2}$; fasting glucose $6.1 \pm 0.7 \mathrm{mmol} / \mathrm{l} ; \mathrm{HbA}_{1 \mathrm{c}} 5.7 \pm 0.4 \%$ ) undergoing elective surgery. One obese subject received concomitant diabetes 


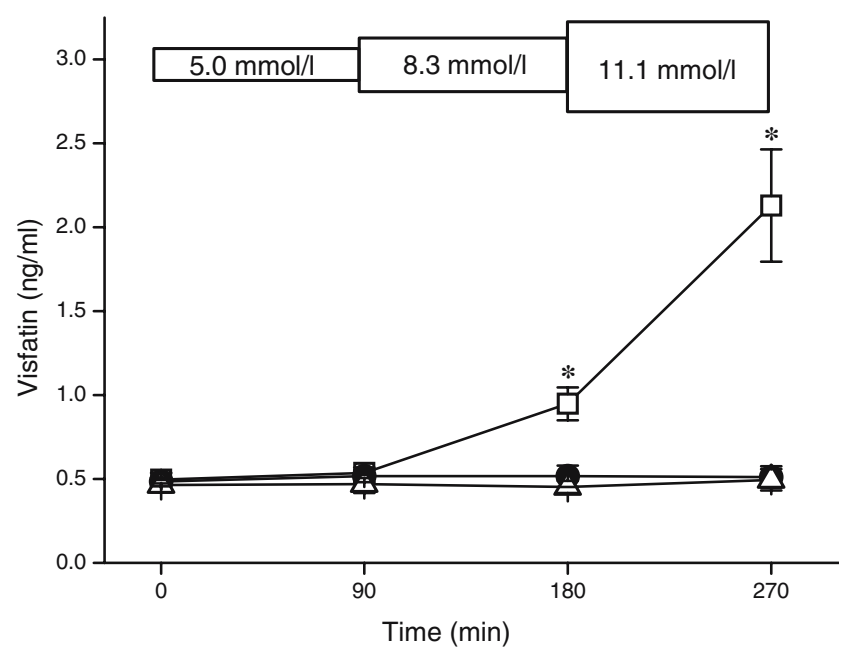

Fig. 1 Visfatin plasma concentration during a glucose clamp over $270 \mathrm{~min}$ with stepwise increased glucose target concentrations of 5.0, 8.3 and $11.1 \mathrm{mmol} / 1$ (see boxes) for a period of $90 \mathrm{~min}$ each. Coinfusion of insulin (solid circles) or somatostatin (open triangles) prevented the increase in visfatin concentrations observed during placebo control experiments (open squares). Means $\pm \mathrm{SD}(n=9)$, $* * p<0.01$ vs pre-dose, ANOVA

therapy with metformin; all other subjects were drug-free. Adipocytes were isolated and cultured according to the protocol published by Seboek et al. [12]. In brief, after removal, adipose tissue was transferred into PBS containing $20 \mathrm{mg} / \mathrm{ml} \mathrm{BSA}$. Adipose tissue was dissected from fibrous material and blood vessels and was digested for $90 \mathrm{~min}$ in PBS containing $20 \mathrm{mg} / \mathrm{ml} \mathrm{BSA}$ and $250 \mathrm{U} / \mathrm{ml}$ Clostridium histolyticum collagenase (Biochrom, Berlin, Germany). The dispersed tissue was filtered through nylon mesh (pore size $150 \mu \mathrm{m})$ and was centrifuged for $10 \mathrm{~min}$ at $200 \mathrm{~g}$. Sedimented cells were resuspended in erythrocyte-lysis buffer containing $0.154 \mathrm{~mol} / 1 \mathrm{NH}_{4} \mathrm{Cl}, 10 \mathrm{mmol} / 1 \mathrm{KHCO}_{3}$ and $0.1 \mathrm{mmol} / \mathrm{l}$ EDTA for $10 \mathrm{~min}$. The cells were then washed with PBS and resuspended in DMEM/Nutrient Mix F12 (DMEM/F12) (1:1) containing $1 \mathrm{mmol} / \mathrm{l} \mathrm{HEPES} \mathrm{and}$ $2.5 \mathrm{mmol} / \mathrm{l}$ L-glutamine (Life Technologies, Karlsruhe, Germany) supplemented with $1.125 \mathrm{~g} / 1 \mathrm{NaHCO}_{3}, 10 \%$ fetal calf serum and $50 \mu \mathrm{g} / \mathrm{ml}$ gentamicin. Cells were seeded in six-well culture plates (Becton Dickinson, Heidelberg, Germany) at a density of 150,000 cells/well. On the following day, when most cells were attached to the plates and after washing twice with PBS, stromal cells were further cultured in a defined serum-free medium to induce differentiation into adipocytes. The adipogenic medium consisted of DMEM/F12 supplemented with $1.125 \mathrm{~g} / 1$ $\mathrm{NaHCO}_{3}, 50 \mu \mathrm{g} / \mathrm{ml}$ gentamicin, $10 \mu \mathrm{g} / \mathrm{ml}$ transferrin, $100 \mathrm{nmol} / 1$ cortisol, $66 \mathrm{nmol} / 1$ insulin and $200 \mathrm{pmol} / 1$ triidothyronine. During the first 2 days, $20 \mu \mathrm{mol} / 1$ 3-isobutyl-1-methylxanthine was also added to the adipogenic medium. Medium was exchanged every $48 \mathrm{~h}$. Within 16 days of culture, stromal preadipocytes differentiated into adipocytes, which were grown to $70-80 \%$ confluence. All buffers and media were adjusted to $\mathrm{pH}$ 7.4.

After $24 \mathrm{~h}$ of resting, cells were incubated with or without glucose at different concentrations $(5.0,8.3$ and $11.1 \mathrm{mmol} / \mathrm{l}$ ) for up to $4 \mathrm{~h}$. Further co-incubations with standard concentrations of insulin (600 pmol/l) [13] and the phosphatidylinositol 3-kinase (PI3-kinase) inhibitor wortmannin (100 nmol/l) [14], protein kinase B (AKT) inhibitor (10 $\mu \mathrm{mol} / \mathrm{l})$ [15] (both Alexis, Lausen, Switzerland) and insulin-receptor antibody (20 nmol/1, Ab 3; Labvision, Fremont, CA, USA) [16] were tested. Supernatants of the culture media were stored and analysed for visfatin concentrations.

Quantitative visfatin mRNA analyses

Total adipocyte mRNA was prepared using the Amp RNA easy kit (Qiagen, Valencia, CA, USA), directly transcribed into cDNA using the RT-Reagent kit and stored at $-80^{\circ} \mathrm{C}$ until analysis. Real-time PCR was performed in 96-well optical plates (Applied Biosystems, Foster City, CA, USA). The $25-\mu 1$ reaction in each well contained $2.5 \mu$ l of the Quantitative PCR Core Reagent Kit (Qiagen) and $1 \mu$ total cDNA in $1 \times$ Taqman Universal PCR master mix (Applied Biosystems). Controls without and with a template of known amplification were included in each assay. The reaction was performed in a sequence detection system (ABI PRISM 7700; Applied Biosystems). For analysis of SYBR, a Green Assay Reagents kit was purchased from Applied Biosystems. The primer sequence for human visfatin used was: $5^{\prime}$-gacgccagcagggaattttgttac-3' (sense) and $5^{\prime}$-agcttttgtcaccttgccattct-3' (antisense) [7].

\section{Statistical analysis}

Because of the skewed distribution of the parameters under study, non-parametric tests were used. Between-group comparisons were performed using the Mann-Whitney $U$-test. Within groups, effects were tested by Friedman's ANOVA and the Wilcoxon signed-rank test. All calculations were performed using a Statistica software package (release 5.1; StatSoft, Tulsa, OK, USA). $p \leq 0.05$ was considered significant. Values are expressed as means \pm SD unless indicated otherwise.

\section{Results}

Clamp studies

Fasting visfatin concentrations were comparable between study days and were $0.5 \pm 0.0 \mathrm{ng} / \mathrm{ml}$ in pooled-data analysis. Glucose infusion caused an increase in circulating insulin 


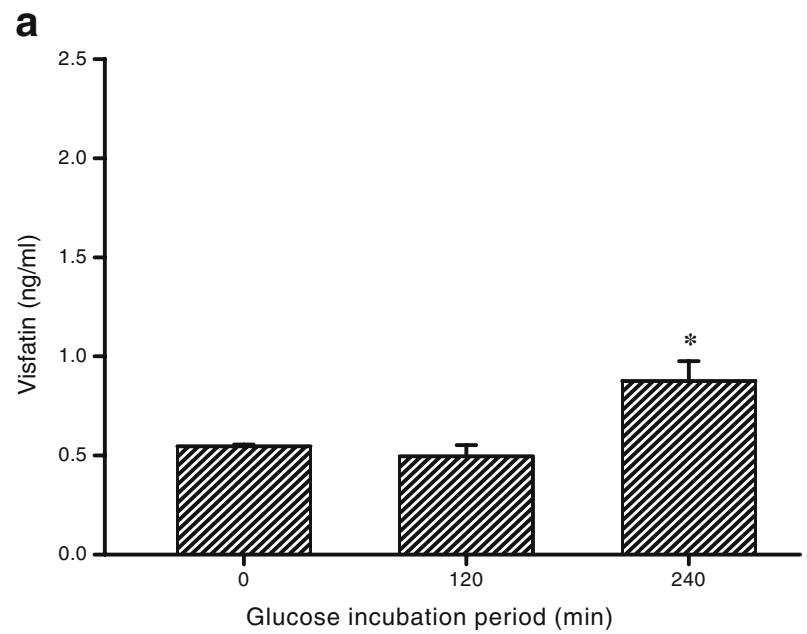

b

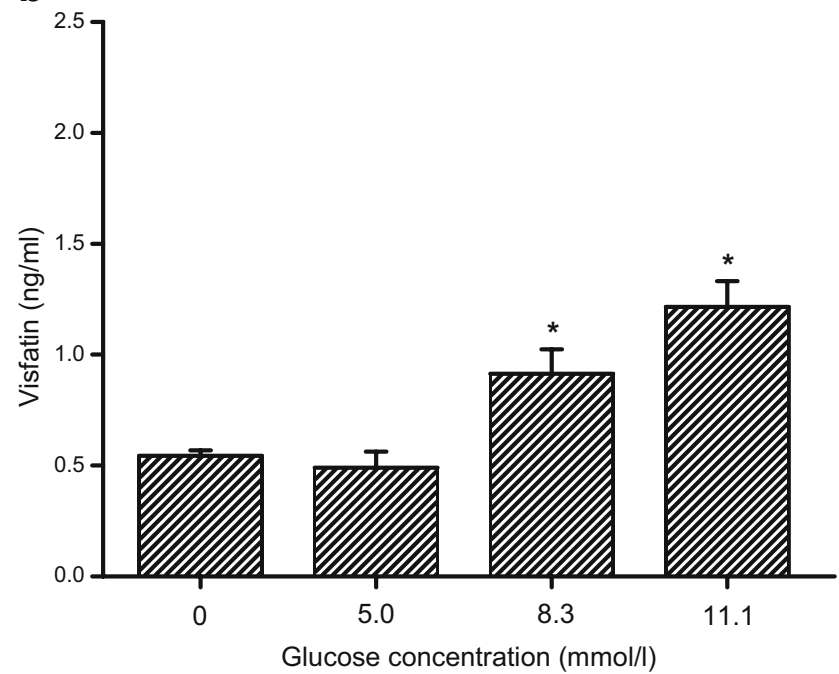

Fig. 2 Visfatin release from cultured human subcutaneous adipocytes from lean patients. Concentrations of visfatin in supernatant media were increased by prolonged glucose exposure at $11.1 \mathrm{mmol} / \mathrm{l}$ (a) and depended on glucose concentration during a 240-min incubation period (b). Means $\pm \mathrm{SD}(n=3),{ }^{*} p<0.05$ vs controls

from $69 \pm 13 \mathrm{pmol} / 1$ to a maximum of $612 \pm 85 \mathrm{pmol} / 1$ $(p<0.01)$ and substantially increased visfatin concentrations from $0.5 \pm 0.0$ to $0.9 \pm 0.1$ and $2.1 \pm 0.3 \mathrm{ng} / \mathrm{ml}$, respectively $(p<0.01$, Fig. 1). Exogenous hyperinsulinaemia had no effect on basal visfatin concentrations but completely prevented the glucose-induced increase of visfatin (Fig. 1). Somatostatin co-administration preserved low fasting insulin concentrations of between $98 \pm 25 \mathrm{pmol} / 1$ at baseline and $89 \pm 49 \mathrm{pmol} / \mathrm{l}$ during glucose infusion. Basal visfatin concentrations of $0.5 \pm 0.0 \mathrm{ng} / \mathrm{ml}$ remained unchanged during clamp studies in the presence of somatostatin (Fig. 1).

Isolated adipocytes

Incubation with glucose caused a time- and dose-dependent visfatin release from isolated subcutaneous adipocytes from

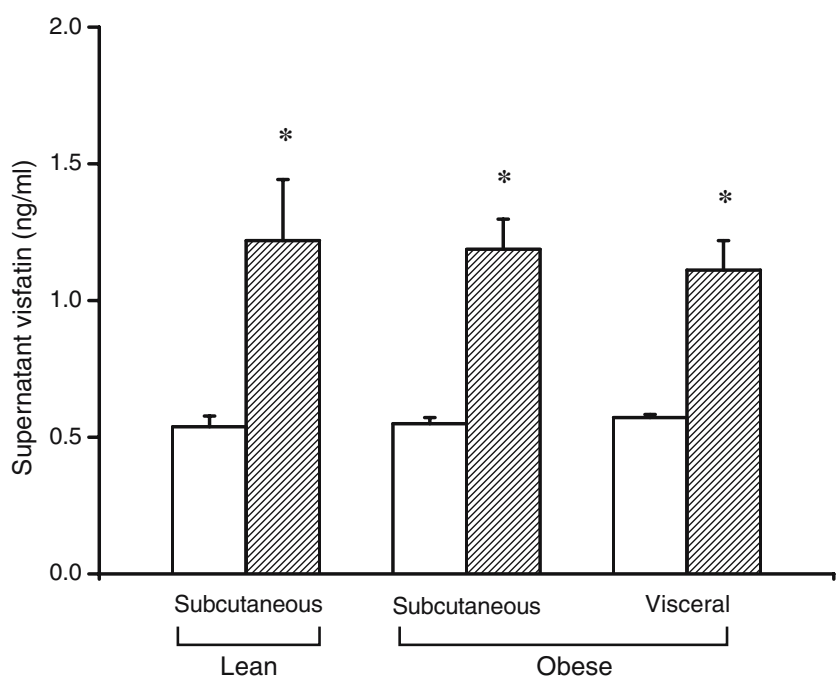

Fig. 3 Visfatin release from subcutaneous and visceral adipocytes from lean $(n=3)$ and obese $(n=4)$ subjects. Adipocytes were incubated without (open columns) or with (hatched columns) $11.1 \mathrm{mmol} / 1$ glucose for 240 min. Means $\pm \mathrm{SD}, * p<0.05$ vs incubation without glucose

lean subjects. Glucose concentrations of $11.1 \mathrm{mmol} / 1$ significantly increased supernatant visfatin concentrations by approximately $50 \%$ after $4 \mathrm{~h}(p<0.05)$, but not after $2 \mathrm{~h}$ (Fig. 2). Visfatin release was significantly enhanced by incubation with glucose at 8.3 and $11.1 \mathrm{mmol} / 1$ compared with control and physiological glucose concentrations of $5.0 \mathrm{mmol} / 1 \quad(p<0.05$, Fig. 2). Glucose-induced visfatin secretion was comparable between subcutaneous adipocytes isolated from lean and obese subjects and there was no difference between visfatin release from subcutaneous or visceral adipocytes from obese patients (Fig. 3). Incubation with insulin had no effect on supernatant visfatin (Fig. 4), but the presence of insulin suppressed glucose-induced visfatin release (Fig. 4). A similar inhibition was also achieved when glucose was co-incubated with PI3-kinase or AKT inhibitors (Fig. 4). The inhibitors alone had no effect on visfatin release (not shown). Blockade of the insulin receptor using a specific antibody had no effect on the action of glucose or insulin alone (Fig. 5). In contrast, insulin-receptor blockade abrogated the mitigating effect of insulin on glucose-induced visfatin release from adipocytes (Fig. 5). Again, this response pattern was similar in cells from subcutaneous and visceral origin (data not shown). In control experiments, mRNA expression of visfatin in visceral adipocytes $(n=3)$ increased between 16 - and 26fold after a 4-h incubation with $11.1 \mathrm{mmol} / \mathrm{l}$ glucose compared with control conditions. Consistent with protein concentrations, co-incubation with insulin, PI3-kinase or AKT inhibitors prevented this effect of glucose and resulted in a mitigated mRNA increase of 3- to 6-fold, 3- to 8-fold and 3 - to 8-fold, respectively. Again, insulin alone had no effect on visfatin mRNA expression. 


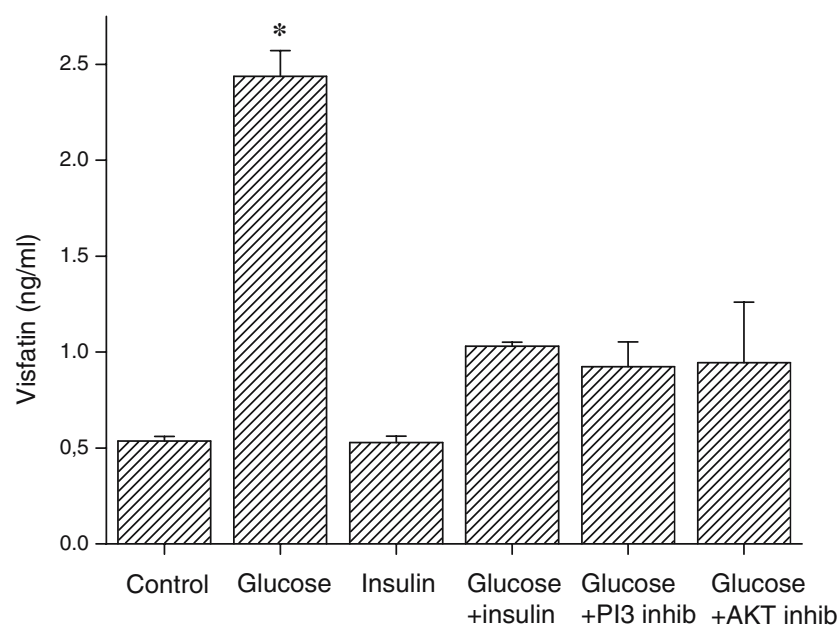

Fig. 4 PI3-kinase- and AKT-dependent regulation of visfatin in cultured subcutaneous adipocytes from lean subjects. Glucose-induced increases of visfatin release were inhibited by co-incubation with insulin, PI3-kinase inhibitor (PI3 inhib), or AKT inhibitor (AKT inhib). Means $\pm \mathrm{SD}(n=3), * p<0.05$ vs control

\section{Discussion}

This study demonstrates that visfatin is released in humans under resting conditions. Basal plasma visfatin concentrations are not altered by hyperinsulinaemia or somatostatin, which suppressed pancreatic insulin excretion during clamp studies. In contrast, hyperglycaemia substantially increased plasma visfatin concentrations, which was prevented by hyperinsulinaemia or somatostatin.

Studies on 3T3-L1 adipocytes have demonstrated that visfatin is regulated by hormones and cytokines influencing glucose homeostasis $[17,18]$. A surprising finding of our

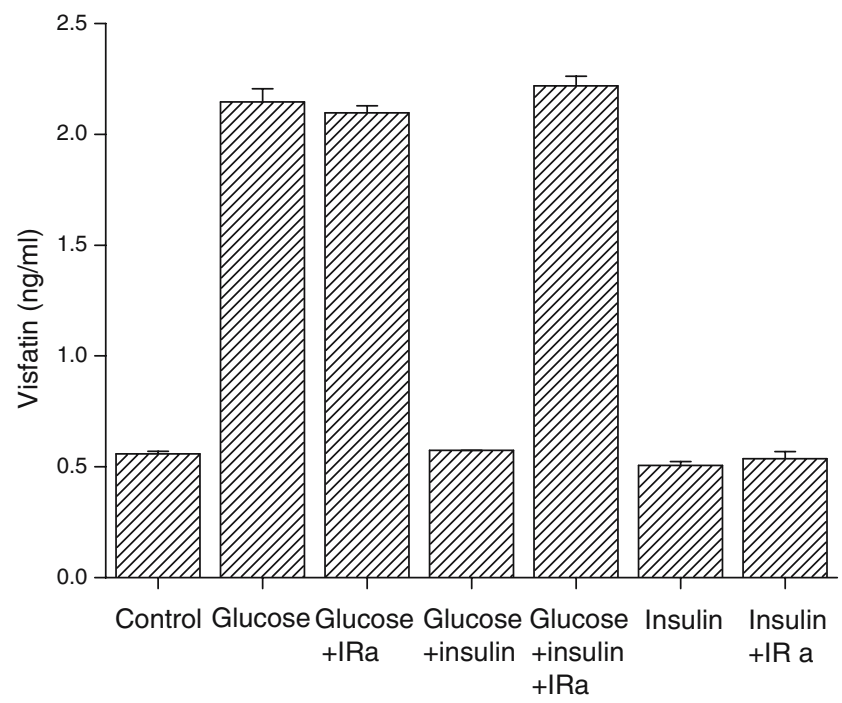

Fig. 5 The glucose-induced visfatin release from cultured subcutaneous adipocytes from lean subjects was not affected by co-incubation with an insulin-receptor antagonist $(I R a)$. In contrast, the inhibitory effect of insulin on glucose-induced visfatin release was prevented by the presence of IRa. Insulin alone did not affect basal visfatin release from adipocytes. Means \pm SD $(n=3)$ studies was the negative feedback of insulin on glucoseinduced visfatin release. The in vitro experiments using a specific insulin-receptor antibody suggest that this effect is directly mediated via insulin-receptor stimulation. Based on animal studies, where blood-glucose-lowering effects of exogenous visfatin were clearly demonstrated [6], one would have expected that visfatin might act synergistically with insulin on the insulin receptors. In cultured adipocytes there was little if any impact of glucose on visfatin release when insulin was present and this effect was fully restored by insulin-receptor blockade. This is consistent with findings from other studies demonstrating that insulin alone had no effect on unstimulated 3T3-L1 adipocytes [17]. Although the physiological actions of visfatin are as yet poorly understood, the negative regulation of hyperinsulinaemia may affect circulating visfatin concentrations in patients with diabetes mellitus type 2 or insulin resistance. Interestingly, the susceptibility of adipocytes to release visfatin upon incubation with glucose in vitro differed substantially between adipocyte cultures. Whether this difference is due to methodological aspects or related to subject characteristics is unknown. The reactivity to hyperglycaemia and hyperinsulinaemia in vivo was, however, homogeneous in the lean subjects under study.

The release of visfatin by adipocytes was dependent on duration and magnitude of glucose elevation. It may therefore be speculated that visfatin plasma concentrations seen in healthy humans during comparatively short clamp conditions may be substantially different from subjects exposed to hyperglycaemia for a prolonged period. Thus, in addition to its relationship with visceral fat, visfatin quantification may also serve as a marker of glucose homeostasis. This is supported by reports demonstrating that visfatin expression and release is increased in obese subjects and in patients with type 2 diabetes mellitus [7, 8] where plasma concentrations are substantially higher than in healthy young subjects. Plasma visfatin concentrations using this assay are generally $<1.0 \mathrm{ng} / \mathrm{ml}$ in healthy young subjects. However, there are no reference values established in elderly subjects and we cannot exclude that visfatin increases with ageing.

The lack of difference between visceral or subcutaneous cultured adipocytes in visfatin release is consistent with previous findings from other authors [7]. Our data suggest that visfatin release may represent a nutrient sensor of adipocytes. However, generation and clearance of visfatin have not been characterised to date. In particular, the source of plasma visfatin in our experiments is unclear and extrapolation of our results to patients with the metabolic syndrome therefore difficult. Further, it is still being debated whether visfatin synthesis is upregulated in obesity. While some data indicate that visfatin is linked to increments in body weight and development of the metabolic syndrome 
[6], others have failed to detect a relationship between the metabolic syndrome and visfatin [19]. The notion that weight loss after gastric banding surgery is associated with a reduction in circulating visfatin concentrations [9] is compatible with the hypothesis that prolonged metabolic stimulation influences adipocytokine levels.

The effect of glucose on visfatin release from adipocytes depends on the PI3-kinase/AKT pathway. Interestingly, PI3-kinase or AKT inhibition resulted in an approximately equivalent effect against glucose-induced visfatin secretion as co-incubation with insulin alone. This effect of PI3kinase inhibition is consistent with results by others on glucose signal transduction [20,21], and comparable results have been obtained for the activation of AKT [22]. The time required for visfatin release during glucose incubation indicates that upregulation of protein synthesis is an underlying mechanism of elevated plasma concentrations, which was confirmed by visfatin mRNA analysis. On the other hand, hyperglycaemia was maintained over a relatively short period only during clamp studies, which suggests that intracellular visfatin stores may be rapidly secreted. While likely candidates of signal transduction for glucose-induced visfatin release have been described in this study, the detailed mechanisms of protein synthesis upregulation and/or release have to be investigated further.

In summary, basal visfatin release is enhanced by glucose in adipocytes in vitro and by hyperglycaemia in healthy humans. This effect is counterregulated by insulin and somatostatin in vivo. The PI3-kinase/AKT axis represents an important intracellular signalling pathway for the action of glucose in adipocytes.

Acknowledgements We thank C. Fuchs for logistic support and her assistance during clamp studies.

\section{References}

1. Friedman JM, Halaas JL (1998) Leptin and the regulation of body weight in mammals. Nature 395:763-770

2. Maeda K, Okubo K, Shimomura I, Funahashi T, Matsuzawa Y, Matsubara K (1996) cDNA cloning and expression of a novel adipose specific collagen-like factor, apM1 (AdiPose Most abundant Gene transcript 1). Biochem Biophys Res Commun 221:286-289

3. Scherer PE, Williams S, Fogliano M, Baldini G, Lodish HF (1995) A novel serum protein similar to $\mathrm{C} 1 \mathrm{q}$, produced exclusively in adipocytes. J Biol Chem 270:26746-26749

4. Hu E, Liang P, Spiegelman BM (1996) AdipoQ is a novel adipose-specific gene dysregulated in obesity. J Biol Chem 271:10697-10703
5. Steppan CM, Bailey ST, Bhat S et al (2001) The hormone resistin links obesity to diabetes. Nature 409:307-312

6. Fukuhara A, Matsuda M, Nishizawa M et al (2005) Visfatin: a protein secreted by visceral fat that mimics the effects of insulin. Science 307:426-430

7. Berndt J, Klöting N, Kralisch S et al (2005) Plasma visfatin concentrations and fat depot-specific mRNA expression in humans. Diabetes 54:2911-2916

8. Chen MP, Chung FM, Chang DM et al (2006) Elevated plasma level of visfatin/pre-B cell colony-enhancing factor in patients with type 2 diabetes mellitus. J Clin Endocrinol Metab 91:295-299

9. Haider DG, Schindler K, Schaller G, Prager G, Wolzt M, Ludvik B (2006) Increased plasma visfatin concentrations in morbidly obese subjects are reduced after gastric banding. J Clin Endocrinol Metab 91:1578-1581

10. Schaller G, Schmidt A, Pleiner J, Woloszczuk W, Wolzt M, Luger A (2003) Plasma ghrelin concentrations are not regulated by glucose or insulin: a double-blind, placebo-controlled crossover clamp study. Diabetes 52:16-20

11. Kerchner J, Colaluca DM, Juhl RP (1980) Effect of whole blood on insulin adsorption onto intravenous infusion systems. Am J Hosp Pharm 37:1323-1325

12. Seboek D, Linscheid P, Zulewski H et al (2004) Somatostatin is expressed and secreted by human adipose tissue upon infection and inflammation. J Clin Endocrinol Metab 89:4833-4839

13. Sandqvist MM, Eriksson JW, Jansson PA (2001) Increased lactate release per fat cell in normoglycemic first-degree relatives of individuals with type 2 diabetes. Diabetes 50:2344-2348

14. Chang-Hui L, Yen-Ju H, Yin-Chou L (2004) Celecoxib simulates respiratory burst through pertussis toxin-sensitive G-protein, a possible signal for beta 2-integrin expression on human neutrophils. Eur J Pharmacol 484:29-39

15. Myou S, Leff AR, Myo S et al (2003) Activation of group IV cytosolic phospholipase A2 in human eosinophils by phosphoinositide 3-kinase through a mitogen-activated protein kinaseindependent pathway. J Immunol 171:4399-4405

16. Taylor R, Soos MA, Wells A, Argyraki M, Siddle K (1987) Insulin-like and insulin-inhibitory effects of monoclonal antibodies for different epitopes on the human insulin receptor. Biochem J 242:123-129

17. Kralisch S, Klein J, Lossner U et al (2005) Hormonal regulation of the novel adipocytokine visfatin in 3T3-L1 adipocytes. J Endocrinol 185:R1-R8

18. Kralisch S, Klein J, Lossner $U$ et al (2005) Interleukin-6 is a negative regulator of visfatin gene expression in 3T3-L1 adipocytes. Am J Physiol Endocrinol Metab 289:E586-E590

19. Klöting N, Klöting I (2005) Visfatin: gene expression in isolated adipocytes and sequence analysis in obese WOKW rats compared with lean control rats. Biochem Biophys Res Commun 332:1070-1072

20. Sautin YY, Lu M, Gaugler A, Zhang L, Gluck SL (2005) Phosphatidylinositol 3-kinase-mediated effects of glucose on vacuolar $\mathrm{H}^{+}$-ATPase assembly, translocation, and acidification of intracellular compartments in renal epithelial cells. Mol Cell Biol 25:575-589

21. Tardif A, Julien N, Chiasson JL, Coderre L (2003) Stimulation of glucose uptake by chronic vanadate pretreatment in cardiomyocytes requires PI 3-kinase and p38 MAPK activation. Am J Physiol Endocrinol Metab 284:E1055-E1064

22. Yaworsky K, Somwar R, Ramlal T, Tritschler HJ, Klip A (2000) Engagement of the insulin-sensitive pathway in the stimulation of glucose transport by alpha-lipoic acid in 3T3-L1 adipocytes. Diabetologia 43:294-303 